\title{
Évaluation du potentiel acaricide des monoterpènes et leurs mélanges binaires sur l'acarien Tetranychus urticae Koch (Acari : Tetranychidae)
}

\author{
Manuscrit reçu le 16 décembre 2020 et accepté le 12 février 2021
}

Asmae BAKKALi AissaOUI ${ }^{1 *}$, Said ZANTAR ${ }^{2}$, Amal El AMrani ${ }^{1}$

\begin{abstract}
${ }^{1}$ Université Abdel Malek Essaadi. Faculté des Sciences et Techniques de Tanger. Département des Sciences de la Vie. Equipe Biochimie et Génétique Moléculaire, BP. 416. MA-Tanger (Maroc).

${ }^{2}$ Institut National de la Recherche Agronomique Unité de la Recherche sur les Techniques Nucléaires, l'Environnement et la Qualité, MA-Tanger (Maroc).
\end{abstract}

\section{Résumé}

Tetranychus urticae Koch (Acari: Tetranychidae) est un acarien polyphage, reconnu comme un ravageur majeur pour une grande quantité de plantes cultivées. Cet acarien cause d'importants dégâts sur les cultures de fraises dans la région du Loukkos au nord du Maroc, se traduisant par de lourdes pertes économiques. L'objectif de ce travail est de mettre en évidence les propriétés acaricides de six monoterpènes purs et de leurs mélanges binaires artificiels sur l'acarien tétranyque à deux points $T$. urticae Koch puis d'analyser les interactions qui existent entre ces composés monoterpéniques. Les monoterpènes testés sont: le thymol, le 1,8-cinéole, le camphre, le linalool, le carvacrol et l' $\alpha$-pinène. Les monoterpènes à l'état pur ou en mélange binaire $(1: 1)$ sont appliqués par contact ou par inhalation sur les acariens adultes à des doses comprises entre 0,5 et $8 \%$.

Nos résultats indiquent que les monoterpènes et leurs mélanges présentent des niveaux de toxicité variables en fonction du mode de leur application, de la dose utilisée et des constituants combinés. Les monoterpènes appliqués par inhalation sont généralement plus toxiques que ceux appliqués par contact. Les mélanges binaires sont généralement plus efficaces que les monoterpènes testés individuellement ce qui indique un effet additif ou synergique entre les composés monoterpéniques combinés. Parmi les combinaisons testées, une seule présente un effet antagoniste (1,8-cinéole et $\alpha$-pinène). Les résultats du présent travail permettent de conclure que certains monoterpènes pourraient être sélectionnés pour développer des mélanges naturels pouvant servir pour lutter contre $T$. urticae, ce qui présente une alternative très prometteuse aux pesticides synthétiques.

Mots-clés. Tetranychus urticae, activité acaricide, monoterpènes, mélanges binaires, interactions.

\footnotetext{
*Adresse pour le courrier électronique : asmaebakkaliaissaoui@gmail.com
} 


\begin{abstract}
:
Tetranychus urticae koch (Acari: Tetranychidae) is a polyphagous mite, recognized as a major pest for a large number of cultivated plants. This mite is causing extensive damage to strawberry crops in the Loukkos region of northern Morocco, resulting in heavy economic losses. The objective of this work is to evaluate the acaricidal properties of six pure monoterpenes and their binary artificial blends on the two spotted spider mite T.urticae and then to analyze the interactions between these monoterpene compounds. The monoterpenes tested are: thymol, 1,8-cineole, camphor, linalool, carvacrol and $\alpha$-pinene. The pure monoterpenes are applied individually or binary artificial blend $(1: 1)$ by contact and fumigation against the adult mites at doses between 0.5 and $8 \%$.

Our results indicate that monoterpenes and their mixtures exhibit varying levels of toxicity depending on the mode of their application, the dose used and the constituents combined. Monoterpenes applied by fumigation are generally more toxic than those applied by contact. Binary blends are generally more effective than monoterpenes tested individually indicating an additive or synergistic effect between the combined monoterpene compounds. Among the combinations tested, only one exhibits an antagonistic effect (1,8-cineole and $\alpha$-pinene). The results of this work allow us to conclude that some monoterpenes could be selected to develop natural blends that can be used to control T. urticae, which presents a very promising alternative to synthetic pesticides.
\end{abstract}

Keywords: Tetranychus urticae, acaricide activity, monoterpenes, binary blends, interactions.

\title{
1. Introduction
}

Au Maroc, la production de fraise dans la zone du Loukkos a connu une croissance spectaculaire depuis le début des années 90 (Lagziri, 2013). Toutefois, l'utilisation intensive de certaines pratiques agricoles représente un obstacle majeur qui entrave cette dynamique de croissance, en terme de durabilité de la production agricole (Lagziri et al., 2015). La culture de la fraise fait actuellement l'objet de traitements phytosanitaires intensifs dans le but de contrôler les nombreux ravageurs qui l'affectent. Parmi ceux, les insecticides de la famille des pyréthrinoïdes de synthèse (comme la lambda-cyhalothrine), les fongicides tel que le mancozèbe de la famille des dithiocarbamates et l'hexaconazole de la famille des triazoles, et les acaricides tel que l'abamectine, un acaricide spécifique de la famille des avermectines, introduit il y a quelques années dans les programmes de contrôle des acariens dans les cultures de fraises, au Nord du Maroc (Lagziri et al., 2015).

L'acarien ravageur Tetranychus urticae Koch (Acari, Tetranychidae) est considéré parmi les ravageurs les plus redoutables des cultures de fraise (Marquier \& Arnault, 2008). Cette espèce est responsable d'importantes pertes de récolte. Son contrôle nécessite des investissements économiques et environnementaux de grande importance. La pullulation de ce ravageur est due à l'utilisation intensive et continue de produits phytosanitaires organiques de synthèse peu sélectifs, comme actuellement signalé dans la vallée marocaine du Loukkos. L'emploi de 
produits phytosanitaires non sélectifs et très persistants peut générer de nombreux problèmes tel l'apparition de résistances (comme celle enregistré contre le Chlorfenapyr (Herron \& Rophail, 2003 ; Van Leeuwen et al., 2004), le Fenpyroximate (Kim et al., 2004) et le Pyridaben (Kim et al., 2006), la pullulation rapide des ravageurs, la pollution de l'environnement et la présence de résidus toxiques, en particulier sur les végétaux traités. Certains acaricides (Le Chlorpyrios de la famille des organo-phosphorés, le Fipronil de la famille de Phénypyrazol et le Carbofuran de la famille de Carbamate) ont été interdits à cause du grand danger que peut entraîner leur utilisation sur la santé humaine (Rabiou, 2019). Devant cette situation, il est primordial de développer une stratégie de lutte contre les ravageurs agricoles, basée non sur l'emploi de pesticides chimiques mais plutôt sur des méthodes alternatives moins polluantes et plus efficaces à long terme. La recherche de nouvelles méthodes utilisant des agents de lutte à faible répercussion écologique s'est beaucoup développée ces dernières années (Agboyi et al., 2009 ; Agboyi 2009 et 2006 ; Attia et al., 2013). Le choix de nouveaux produits alternatifs est guidé par la non-persistance de ces produits et de leurs dérivés dans l'environnement et la faible toxicité de leurs résidus dans les cultures traitées. À l'heure actuelle, une attention particulière est donnée aux produits dérivés de plantes et aux biopesticides d'origine botanique. Le développement de nouveaux biopesticides d'origine botanique revêt un intérêt considérable.

Les huiles essentielles extraites de plantes représentent une véritable richesse et peuvent être à l'origine d'un grand nombre de substances acaricides et insecticides exploitables dans le contrôle des ravageurs (Papachristos et al., 2004 ; Ketoh et al., 2006 ; Attia et al. 2011, 2012, 2013). Les propriétés insecticides de nombreuses huiles essentielles sont principalement attribuées aux monoterpènes qui sont généralement volatiles et lipophiles et qui peuvent rapidement pénétrer dans les insectes et interférent avec leurs fonctions physiologiques (Reis et al., 2014). Cette activité peut s'expliquer par la présence de composés majoritaires des huiles essentielles (Carovic-Stanko et al., 2010; Herman et al., 2016). Toutefois, ce ne sont pas uniquement les composés majoritaires des huiles essentielles qui sont responsables de cette activité insecticide, mais il peut y avoir aussi d'autres composés minoritaires qui peuvent interagir d'une façon synergique et antagoniste (Ngamo \& hance, 2007 ; Deletre et al., 2015). Il est, de ce fait, difficile de lier les activités insecticides et répulsives des huiles uniquement à l'effet individuel des constituants de l'huile essentielle; de telles activités pourraient être dues à l'effet synergique de plusieurs éléments de l'huile. Des interactions complexes peuvent se produire entre les constituants majeurs et mineurs d'une manière synergique qui affecte l'activité insecticide/acaricide.

Les composants monoterpéniques présents dans les HEs tels que le thymol, la carvacol, le linalool, le citronellol, le limonène, l' $\alpha$ - pinène et le $\beta$-pinène ont été largement documentés pour être des composés qui possèdent des activités larvicides et adulticides contre les moustiques (Watanabe et al., 1993), les mouches domestiques (Singh \& Singh, 1991), les insectes ravageurs des produits stockés (Tripathi et al., 2003) et les termites (Zhu et al., 2001). Sur les acariens comme l'acarien ravageur T. urticae, il y avait des travaux qui ont porté sur l'effet des composés terpéniques (Miresmailli et al., 2006 ; Hamdy et al., 2009 ; Attia et al., 2013) 
Dans ce sens, les interactions synergiques entre certains monoterpènes contenus dans les huiles essentielles seraient intéressantes à étudier. La connaissance des effets synergiques positifs ou négatifs des monoterpènes contenus dans les huiles essentielles permettra une compréhension plus profonde de l'efficacité des mélanges de monoterpènes. Ceci peut jouer un rôle important dans le développement de nouveaux insecticides/acaricides botaniques.

Le présent travail s'insère dans cette optique et a pour but d'évaluer l'effet de six composés monoterpéniques sur l'acarien ravageur T. urticae, collecté dans des cultures de fraise de la région du Loukkos, au nord du Maroc. Les effets des six monoterpènes suivants sont testés : thymol, carvacrol, 1,8-cinéole, linalool, $\alpha$-pinène et camphre. Ces monoterpènes sont fréquemment retrouvés dans les huiles essentielles extraites des plantes de la famille des Lamiacées (Bounatirou et al., 2007). Les acariens adultes sont exposés à un traitement par contact et par inhalation moyennant des doses croissantes de chaque composé. L'étude a, de même, porté sur l'évaluation de l'effet acaricide par inhalation des mélanges binaires de ces monoterpènes. Le but est de détecter puis d'analyser les interactions qui existent entre ces composés. Comprendre la contribution de chaque composé individuellement et leurs interactions avec la toxicité globale de ces huiles essentielles est indispensable pour optimiser leur utilisation dans la lutte contre l'acarien ravageur T. urticae.

\section{Matériel et méthodes}

\subsection{Collecte et maintien des acariens}

Les acariens $T$. urticae sont collectés à partir de plantes infestées prélevées dans des fraisières situées à Laouamra (Latitude : 31.79, Longitude : -8.71), dans la région de Larache, au nord du Maroc. L'élevage des acariens est réalisé dans une salle climatisée à une température de $25 \mathrm{C}^{\circ}$, une humidité relative de 50 à $60 \%$ et une photopériode de $16 \mathrm{D} / 8 \mathrm{~L}$. Les acariens sont maintenus en élevage sur des plantes de haricot vert (Phaseolus vulgaris L.) et sans aucune exposition aux pesticides.

\subsection{Les monoterpènes et les combinaisons binaires testées}

Six monoterpènes ont été testés pour leur effet acaricide sur l'acarien ravageur T. urticae. Il s'agit du thymol (98,5\%), 1,8-cinéole (99\%), camphre (996\%), linalool (97\%), carvacrol (98\%), $\alpha$-pinène (99\%). Tous les monoterpènes utilisés proviennent de la société Sigma-Aldrich.

Les effets acaricides des 15 combinaisons binaires suivantes sont comparés : (1,8-cinéolethymol), (1,8-cinéole-camphre), (1,8-cinéole-carvacrol), (1,8-cinéole-linalool), (thymolcamphre), (thymol-linalool), (thymol-carvacrol), ( $\alpha$-pinène-linalool), ( $\alpha$-pinène-carvacrol), $(\alpha$ pinène-thymol), ( $\alpha$-pinène-camphre), ( $\alpha$-pinène-1,8-cinéole), (linalool-camphre), (linaloolcarvacrol) et (carvacrol-camphre). 


\subsection{Tests toxicologiques}

La méthode appliquée est celle dite de concentration séquentielle adoptée par l'IRAC (Insecticide Resistance Action Committee, 2012) pour tester l'efficacité des produits phytosanitaires. Le principe consiste à traiter la population adulte de $T$. urticae avec des préparations à base des monoterpènes. Pour réaliser ces essaies, on a laissé des femelles adultes pondre pendant $48 \mathrm{~h}$. Le développement des œufs retirés a abouti à une population uniformément âgée d'acariens. Tous les tests toxicologiques ont été fait sur des acariens adultes âgés de 10 jours. Pour préparer une solution de monoterpène d'une dose donnée, le volume du monoterpène pur correspondant est mélangé à $0.1 \mathrm{ml}$ de solution de triton $0.1 \%$. Ces préparations présentent des doses croissantes. Les cinq doses croissantes suivantes sont utilisées : $0.5 ; 1 ; 2 ; 4$ et $8 \%$ pour chaque monoterpène ou mélange binaire. Pour chaque dose, cinq répétitions ont été réalisées. Le dénombrement des individus morts est effectué après 24 , 48 et $72 \mathrm{~h}$ du début du test. Les individus qui n'arrivent pas à bouger, après une légère excitation avec un pinceau, sont considérés morts. Les valeurs $\mathrm{LC}_{50}$ (concentrations létales entraînant une mortalité de $50 \%$ de la population traitée) et leurs limites de confiance ont été calculés à l'aide de la méthode de Finney (1971).

Les témoins sont traités de la même façon mais avec $0.1 \%$ de triton-X100. Ces témoins permettront de vérifier qu'aucune surmortalité n'était observée et de corriger la mortalité observée selon Abbott (1925).

\subsubsection{Test par contact}

La souche adulte de T. urticae est soumise à une série de bio-essais de toxicité par contact. La méthode adoptée consiste à utiliser des feuilles fraiches du haricot, appliquées sur du papier filtre humidifiée. La feuille du haricot repose sur la face supérieure, sur la boite de pétri $(10 \mathrm{~cm})$ (Overmeer,1967). Cinq adultes de T. urticae sont déposées sur la face inférieure de chaque disque de feuille du haricot. Des préparations à base des monoterpènes testés sont pulvérisées sur les individus de T. urticae avec un pulvérisateur manuel (Butt \& Goettel, 2000). La quantité appliquée est préalablement mesurée et elle est de $9.6 \pm 0.83 \mu 1 / \mathrm{cm}^{2}$. La distance entre le pulvérisateur et la feuille du haricot est estimée à $2 \mathrm{~cm}$.

\subsubsection{Test par inhalation}

Dans chaque bocal en verre, une boule de coton est suspendue à l'aide d'un fil fixé à un morceau de scotch double face qui a été pressé fermement contre la face interne du couvercle. Sur des disques de feuille du haricot fraiches appliquées sur du papier filtre humidifiée, cinq adultes de T. urticae sont déposés et sont introduits dans chaque bocal. Les préparations obtenues sont pulvérisées sur les boules du coton suspendu. L'application est réalisée avec un pulvérisateur manuel dont la quantité appliquée est de $9.6 \pm 0.83 \mu 1 / \mathrm{cm}^{2}$. 


\subsection{Méthode d'estimation des interactions entre les monoterpènes}

Les interactions entre six monoterpènes formant 15 mélanges binaires différents ont été analysées selon la méthode décrite par Singh et al (2009).

Les composés sont combinés dans un rapport (1:1). La toxicité du mélange (composé A + composé B) contre les adultes de T. urticae est évalué en choisissant la valeur $\mathrm{LC}_{50}$ du composé le plus toxique après $24 \mathrm{~h}$ contre l'acarien. La valeur $\mathrm{LC}_{50}$ du composé le plus actif parmi les 2 composés d'un mélange est choisie comme concentration pour chacun des constituants du mélange binaire (Hummelbrunner \& Isman, 2001). En parallèle, la toxicité des composés purs est à nouveau déterminée, cette fois en utilisant la $\mathrm{LC}_{50}$ du composé le plus toxique enregistrée chez l'acarien après $24 \mathrm{~h}$ d'exposition aux vapeurs de ces composés. La mortalité observée de la combinaison binaire est ainsi déduite.

Les interactions entre les composés des mélanges binaires ont été analysés moyennant le test du $\boldsymbol{X}^{\mathbf{2}}$ (Khi2). Les mortalités observées ont été comparées avec celles attendues en utilisant la formule décrite par Hummelbrunner et Isman, (2001) comme suit :

$$
\mathrm{E}=\mathrm{Oa}+\mathrm{Ob}(1-\mathrm{Oa})
$$

Où $\mathrm{E}$ est la mortalité attendue dans le mélange, et $\mathrm{Oa}$ et $\mathrm{Ob}$ sont les valeurs en pourcentage, exprimé entre 0 et 1 , des mortalités observées des composés purs $\mathrm{A}$ et $\mathrm{B}$ respectivement, à la concentration létale du composé plus toxique impliqué dans l'échantillon.

Les effets des mélanges ont été classés comme étant soit antagonistes, additifs ou synergiques à l'aide de comparaisons par le test de $X^{2}$ (Pavela, 2015): $X^{2}=(\mathbf{O m}-\mathbf{E})^{2} / \mathbf{E}$

Où $\mathbf{O m}$ est la mortalité observée de la combinaison binaire et $\mathbf{E}$ est la mortalité attendue de la combinaison ; avec $\boldsymbol{X}^{\mathbf{2}}=\mathbf{3 . 8 4}$ (quand ddl=1 et $\mathrm{p}=0.05$ ).

Selon Hummelbrunner \& Isman (2001), la combinaison est dite synergique quand $X^{2}>3.84$ et $\mathrm{O}_{\mathrm{m}}>\mathrm{E}$, la combinaison est dite antagoniste quand $X^{2}>3.84$ et $\mathrm{O}_{\mathrm{m}}<\mathrm{E}$ et la combinaison est dite additive quand $X^{2}<3.84$.

\subsection{Analyses statistiques}

Les pourcentages de mortalité sont corrigés par la formule d'Abbott (Abbott, 1925). Les traitements statistiques réalisés sont effectués grâce à des analyses de variance (ANOVA) à deux facteurs (dose, durée post traitement), suivies par des tests de Tukey, permettant la comparaison des moyennes deux à deux et des analyses à un facteur (monoterpène) pour comparer la toxicité des monoterpènes testés. Les différents traitements statistiques sont réalisés à l'aide du logiciel SPSS 13.0. Les courbes log dose-réponse ont permis de déterminer la $\mathrm{CL}_{50}$ pour les bioessaies selon l'analyse probit (Finney, 1971). Dans tous les cas le seuil de signification retenu est $: \mathrm{p}<0.05$. 


\section{Résultats}

\subsection{Toxicité des monoterpènes testés individuellement sur T. urticae}

Des analyses de variance à un facteur (monoterpène) ont porté sur la mortalité des adultes de T. urticae pour comparer la toxicité des monoterpènes testés. Il s'agit de : thymol, carvacrol, 1,8-cinéole, $\alpha$-pinène, camphre et linalool.

\subsubsection{Par contact}

Les résultats de l'analyse de variance indiquent que les six monoterpènes testés présentent des niveaux de toxicité différents à l'égard de l'acarien ravageur T. urticae $(\mathrm{F}=9.395$; ddl=9 ; $\mathrm{P}<0.05)$. La comparaison des pourcentages de mortalité engendrés par les différents monoterpènes révèle que le carvacrol est le plus toxique. Il est capable d'éliminer plus que les $3 / 4$ des acariens traités $(77.27 \%$ ), suivi de près par le thymol et le linalool qui provoquent des taux de mortalité respectifs de 75.66 et $72.33 \%$. Une deuxième catégorie de monoterpènes est celle dont le niveau de toxicité engendré se situe entre 69 et $61 \%$. À cette catégorie appartiennent : l' $\alpha$-pinène $(66.33 \%)$ et 1,8-cinéole $(61.25 \%)$. Le camphre apparait le moins offensif sur les acariens (46.33\%) (Figure 1).

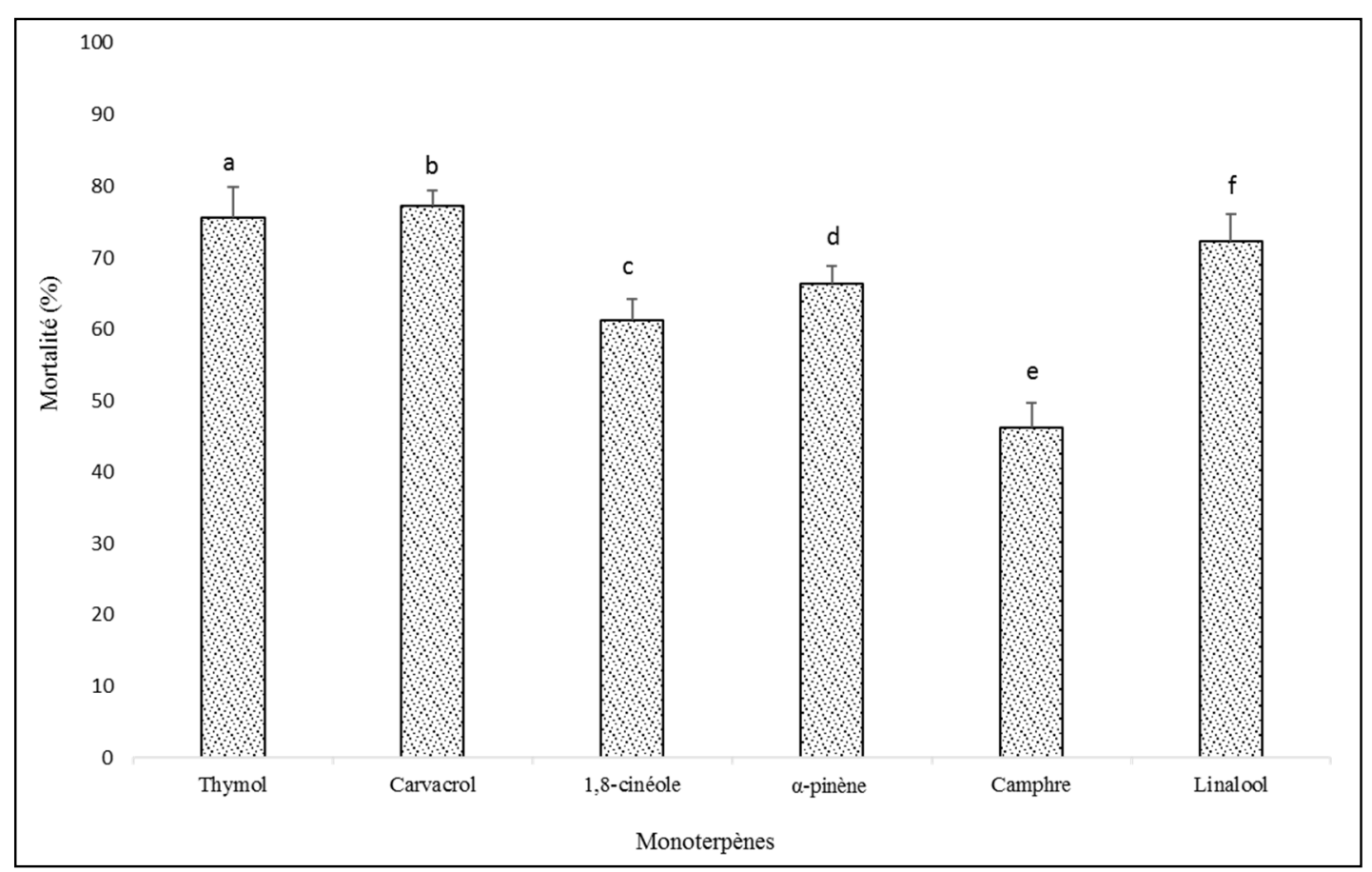

Figure 1 : Comparaison des taux de mortalité des adultes de $T$. urticae traités par contact avec les six monoterpènes testés. Les barres d'erreur représentent les moyennes \pm SEM de cinq répétitions. Les colonnes portant des lettres différentes indiquent qu'il y a une différence significative dans le taux de mortalité entre les monoterpènes selon le test de Tukey HSD, $\mathrm{p} \leq 0,05$. 


\subsubsection{Par inhalation}

L'analyse statistique montre que l'efficacité du traitement par inhalation est variable en fonction $\mathrm{du}$ monoterpène appliqué $(\mathrm{F}=11.525 ; \mathrm{ddl}=9 ; \mathrm{P}<0.05)$. Parmi les monoterpènes testés, le carvacrol s'avère le plus toxique à l'égard des acariens : les taux de mortalité qu'il provoque est de $85 \%$. En seconde position, se classe une seconde catégorie de monoterpènes d'huiles essentielles, capables d'éradiquer entre $3 / 4$ et les $2 / 3$ des acariens traités. Cette catégorie comprend : 1,8-cinéole (77.66\%), le thymol (71.33) et le linalool (69.5\%). La dernière catégorie de monoterpènes est celle ne pouvant éliminer que presque la moitié des acariens traités. À cette catégorie appartiennent : l' $\alpha$-pinène (59.66\%) et le camphre (56.66\%) (Figure 2).

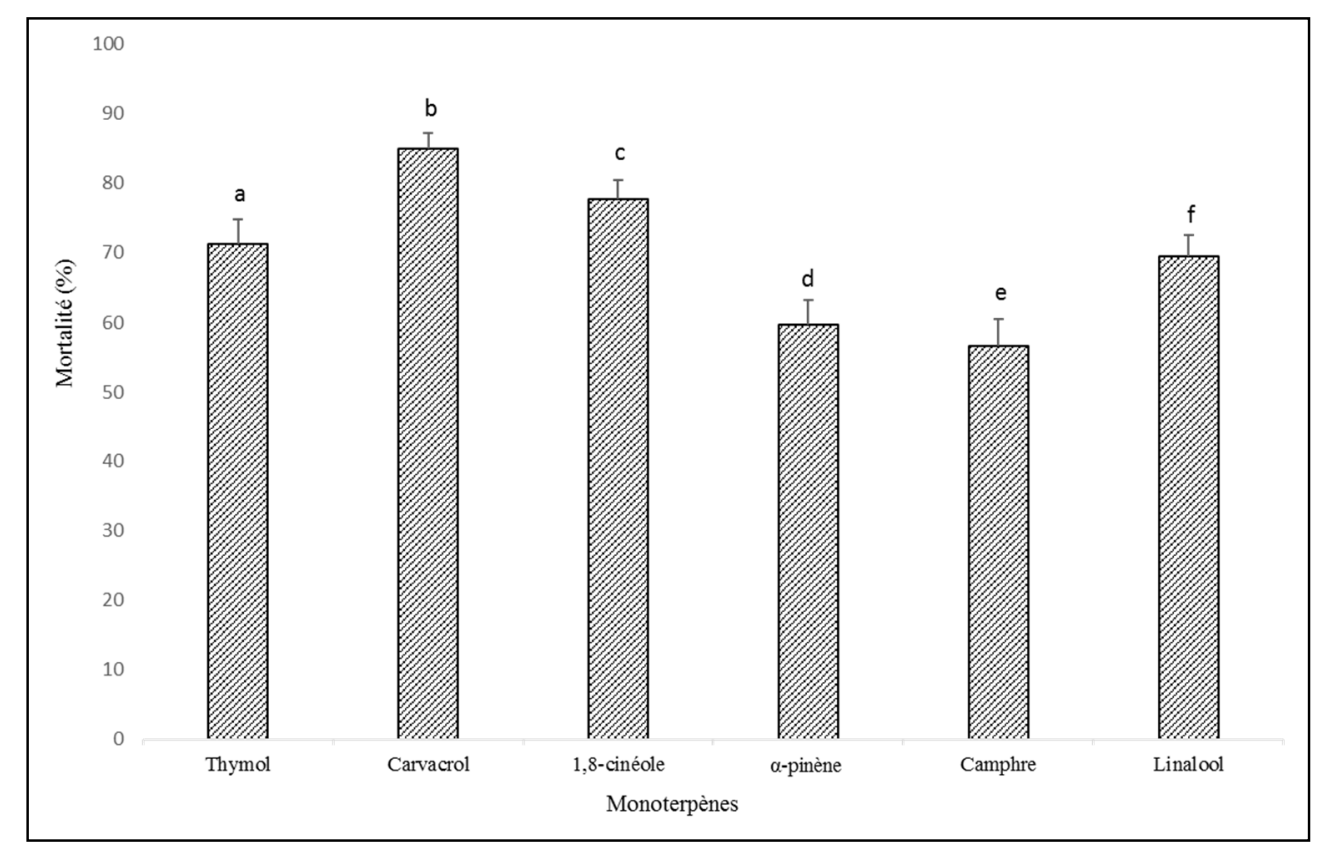

Figure 2 : Comparaison des taux de mortalité des adultes de $T$. urticae traités par inhalation avec les six monoterpènes testés. Les barres d'erreur représentent les moyennes \pm SEM de cinq répétitions. Les colonnes portant des lettres différentes indiquent qu'il y a une différence significative dans le taux de mortalité entre les monoterpènes selon le test de Tukey HSD, $\mathrm{p} \leq 0,05$.

\subsection{Toxicité des mélanges binaires des monoterpènes sur $T$. urticae}

Le traitement par inhalation des acariens avec les différents mélanges binaires de monoterpènes testés révèle les niveaux de toxicité différentiels de ces derniers $(\mathrm{F}=24.53 ; \mathrm{ddl}=14 ; \mathrm{P}<0.05)$. Une létalité totale (100\%) a été observé chez les acariens ayant inhalé les vapeurs des mélanges binaires suivants : (1,8-cinéole-thymol), (1,8-cinéole-carvacrol), (thymol-camphre), (thymollinalool), (thymol-carvacrol), ( $\alpha$-pinène-thymol), (linalool-camphre), (linalool-carvacrol). La seconde catégorie de mélanges binaires de monoterpènes est capable d'éradiquer presque les $3 / 4$ des acariens traités. Il s'agit des mélanges binaires suivants : 1,8-cinéole-linalool (82\%), $\alpha$ - 
pinène-carvacrol (79.5\%), carvacrol-camphre $(77.66 \%)$ et 1,8-cinéole-camphre $(76 \%)$. La dernière classe de mélanges binaires est celle qui a pu éliminer presque les $2 / 3$ des acariens traités. Cette classe regroupe les trois mélanges binaires suivants : $\alpha$-pinène-camphre $(63.33 \%)$, $\alpha$-pinène-linalool (63\%) et $\alpha$-pinène-1,8- cinéole (60\%) (Figure 3).

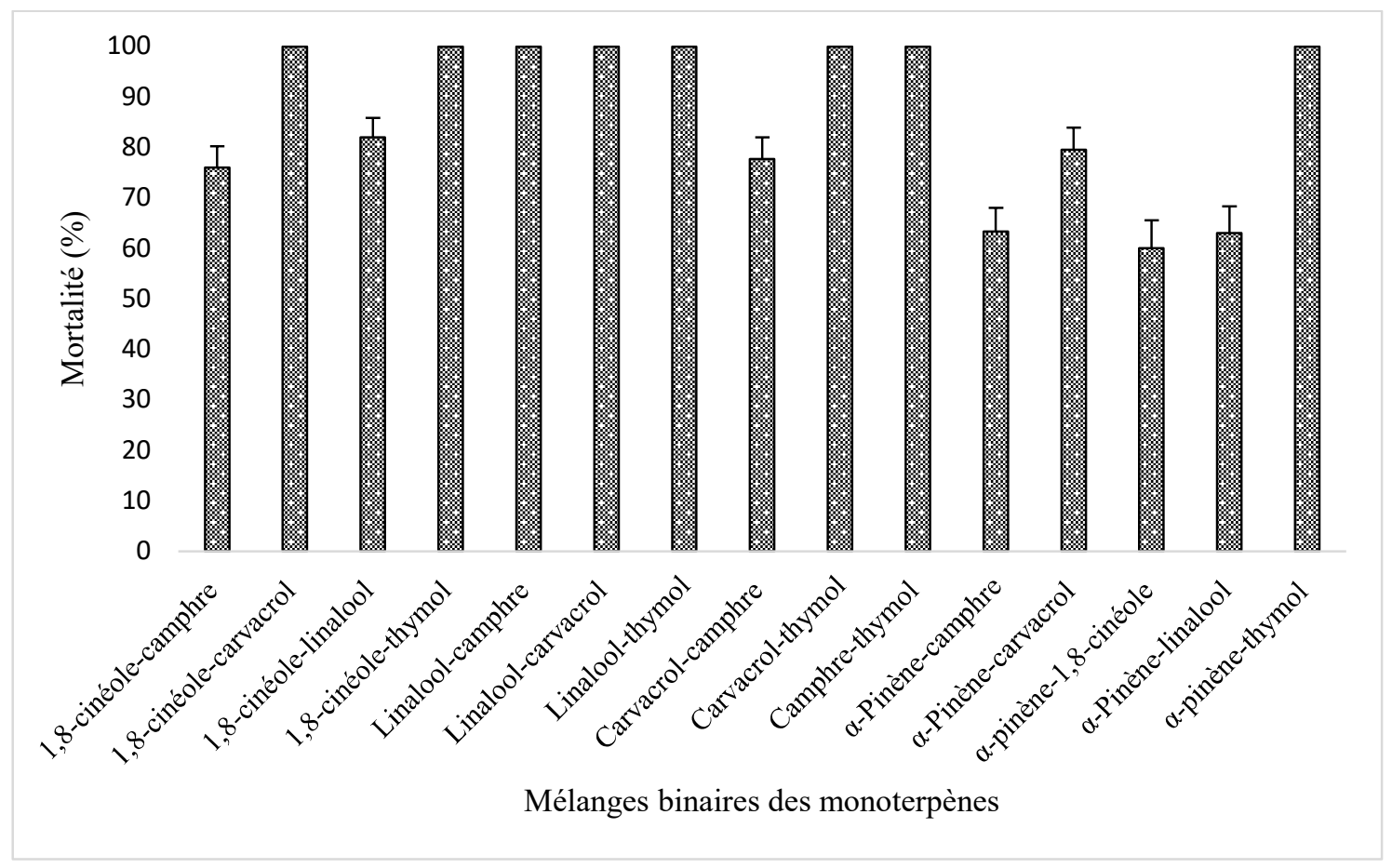

Figure 3 : Comparaison des taux de mortalité des adultes de $T$. urticae traités par inhalation avec les quinze combinaisons des monoterpènes testés. Les barres d'erreur représentent les moyennes \pm SEM de cinq répétitions. Il y a une différence significative dans le taux de mortalité entre les mélanges binaires selon le test de Tukey HSD, $\mathrm{p} \leq 0,05$.

\subsection{Analyse des interactions entre les monoterpènes dans les mélanges binaires testés sur T. urticae}

La formule de Hummelbrunner \& Isman (2001) a été appliquée pour estimer la nature des interactions entre les monoterpènes mélangés dans les solutions binaires préparées. Les résultats obtenus sont décrits dans le tableau ci-dessus (Tableau 1). On montre ainsi que les monoterpènes interagissent entre eux de différentes manières. Les interactions entre les monoterpènes peuvent avoir 3 effets différents: additif, lorsque l'effet létal combiné de deux composés représente la somme des effets de chaque composé pris individuellement (aucune interaction directe), synergique lorsque l'effet létal est plus grand que la somme des effets attendus s'ils avaient opéré indépendamment, ou créent un effet que chacun d'entre eux n'aurait pas créé isolément, ou antagoniste lorsque l'effet combiné d'au moins deux composés est moins toxique que les effets individuels des substances.

Nos résultats montrent que certains monoterpènes présentent des effets additifs comme le cas de thymol et 1,8-cinéole $\left(\boldsymbol{X}^{2}=0.84\right)$. 
D'autres montrent des effets antagonistes comme le cas de 1,8-cinéole et $\alpha$-pinène $\left(X^{2}=10.35\right)$. Une dernière catégorie de monoterpènes sont plutôt synergiques comme le cas thymol et $\alpha$ pinène. Il est à souligner que l'effet d'un monoterpène est variable en fonction du mélange binaire préparé. Autrement dit, il peut avoir un effet additif, synergique ou antagoniste en fonction du second monoterpène qui lui est ajouté dans le mélange binaire. À titre d'exemple, l' $\alpha$-pinène, qui a un effet antagoniste lorsqu'il est mélangé avec 1,8-cinéole, montre plutôt un effet synergique avec le thymol.

Tableau 1. Toxicité des mélanges binaires des monoterpènes sur les adultes de $T$. urticae et les types d'interactions entre les monoterpènes.

\begin{tabular}{|c|c|c|c|c|c|c|c|}
\hline $\begin{array}{c}\text { Monoterpène } \\
\text { A }\end{array}$ & $\begin{array}{c}\text { Monoterpène } \\
\text { B }\end{array}$ & $\begin{array}{l}\text { Mortalité } \\
\text { observée } \\
\text { avec A }\end{array}$ & $\begin{array}{c}\text { Mortalité } \\
\text { observée } \\
\text { avec B }\end{array}$ & $\begin{array}{l}\text { Mortalité } \\
\text { attendue de } \\
\text { mélange } \\
\text { (E) }\end{array}$ & $\begin{array}{l}\text { Mortalité } \\
\text { observée } \\
\text { avec le } \\
\text { mélange } \\
\text { (Om) }\end{array}$ & $X^{2}$ & Effet \\
\hline 1,8-cinéole & Thymol & $75 \pm 2,27$ & $65 \pm 2,9$ & 91,25 & 100 & 0,84 & Additif \\
\hline 1,8-cinéole & Camphre & $75 \pm 2,27$ & $40 \pm 3,676$ & 85 & 95 & 1,17 & Additif \\
\hline 1,8-cinéole & Carvacrol & $75 \pm 2,27$ & $90 \pm 3,014$ & 97,5 & 100 & 0,064 & Additif \\
\hline 1,8-cinéole & Linalool & $75 \pm 2,27$ & $65 \pm 2,76$ & 91,25 & 100 & 0,84 & Additif \\
\hline 1,8-cinéole & $\alpha$-pinène & $75 \pm 2,27$ & $38.33 \pm 4,23$ & 84,6 & 55 & 10,35 & Antagoniste \\
\hline Thymol & Camphre & $65 \pm 2,9$ & $40 \pm 3,676$ & 79 & 100 & 5,58 & Synergique \\
\hline Thymol & Carvacrol & $65 \pm 2,9$ & $90 \pm 3,014$ & 96,5 & 100 & 0,12 & Additif \\
\hline Thymol & Linalool & $65 \pm 2,9$ & $65 \pm 2,76$ & 87,75 & 100 & 1,71 & Additif \\
\hline Thymol & $\alpha$-pinène & $65 \pm 2,9$ & $38.33 \pm 4,23$ & 78,4 & 100 & 5,95 & Synergique \\
\hline$\alpha$-pinène & Camphre & $38.33 \pm 4,23$ & $40 \pm 3,676$ & 63 & 50 & 2,68 & Additif \\
\hline$\alpha$-pinène & Carvacrol & $38.33 \pm 4,23$ & $90 \pm 3,014$ & 93,8 & 100 & 0,4 & Additif \\
\hline$\alpha$-pinène & Linalool & $38.33 \pm 4,23$ & $65 \pm 2,76$ & 78,4 & 100 & 5,95 & Synergique \\
\hline Linalool & Camphre & $65 \pm 2,76$ & $40 \pm 3,676$ & 79 & 100 & 5,58 & Synergique \\
\hline Linalool & Carvacrol & $65 \pm 2,76$ & $90 \pm 3,014$ & 96,5 & 100 & 0,127 & Additif \\
\hline Carvacrol & Camphre & $90 \pm 3,014$ & $40 \pm 3,676$ & 94 & 100 & 0,38 & Additif \\
\hline
\end{tabular}




\section{Discussion}

Le présent travail a porté sur six monoterpènes (thymol, carvacrol, 1.8-cineole, linalool, $\alpha$ pinène et camphre) fréquemment retrouvés dans les huiles essentielles des plantes de la famille des Lamiacées (Bounatirou et al., 2007). Les monoterpènes testés appartiennent aux 2 grandes familles chimiques suivantes: les hydrocarbures monoterpèniques ( $\alpha$-pinène) et les monoterpènes oxygénés. Ces derniers peuvent être des phénols (thymol, carvacrol) ou des alcools (linalool), des éthers (1,8-cinéole) ou des cétones (camphre).

Le premier volet de cette étude consiste à comparer la toxicité des six monoterpènes en fonction de leur mode d'application: directement par contact ou par inhalation. Les composés phytochimiques et les monoterpènes, en particulier, sont reconnus avoir une activité acaricide attribuée au caractère lipophile de leur squelette hydrocarboné et au caractère hydrophile de leurs groupements fonctionnels (Badawy et al., 2010 ; Pandey et al., 2013).

D'une manière générale, nous montrons que les monoterpènes testés sont plus toxiques sur les acariens adultes s'ils sont appliqués par inhalation que s'ils sont appliqués par contact. Des observations similaires ont été faites par Kim et al (2003) qui ont noté aussi que les monoterpènes présentent une activité acaricide par inhalation plus importante que par contact, en raison de leurs effets plus importants sur le système respiratoire de l'acarien.

Cette constatation a été confirmée par Badawy et al (2010) qui, grâce au modèle QSAR (Structure-Activité Quantitative Relationnelle), permettant la prédiction des activités de nouveaux produits chimiques, ont trouvé un coefficient positif de la pression de vapeur des monoterpènes. Ces auteurs ont étudié aussi la solubilité des composés, qui est une autre composante des prédictions QSAR. Ils ont montré une augmentation de l'hydrophobicité qui se traduit par une diminution de l'activité acaricide des monoterpènes. Les résultats suggèrent que ces composés sont largement délivrés en phase vapeur et que leur toxicité dépend de l'accès via le système respiratoire (Badawy et al., 2010). Cependant, la nature précise de leurs modes d'action reste à clarifier.

D’une façon générale, dans le cas de l'application par contact, les monoterpènes oxygénés : carvacol et thymol (phénols) et linalool (alcool) dépassent par leur toxicité les hydrocarbures monoterpéniques. Une exception est, toutefois, faite pour le 1,8-cinéole (éther) et pour le camphre (cétone), deux monoterpènes oxygénés qui présentent une toxicité par contact moins importante que certains hydrocarbures monoterpéniques. Cette constatation a été prouvé par certains auteurs qui ont rapporté une efficacité plus forte des monoterpènes appartenant aux hydrocarbures que ceux des alcools, des aldéhydes et des cétones (Dias \& Moraes, 2014 ; Seo et al., 2015) ce qui laisse suggérer l'intervention d'autres facteurs et d'autres descripteurs dans la modulation de la toxicité d'un composé donné. Dans un ordre décroissant la toxicité par contact des monoterpènes oxygénés se classent de la façon suivante : carvacol (phénol) > thymol (phénol) $>$ linalool (alcool) $>1,8$-cinéole (éther) $>$ camphre (cétone).

Dans le cas de l'application par inhalation aussi, les monoterpènes oxygénés présentent, d'une façon générale, une plus grande toxicité par rapport aux non oxygénés. La toxicité inhalatoire 
des monoterpènes oxygénés peut se classer de la façon suivante : Carvacrol (phénol) > 1,8cinéole (éther) $>$ thymol (phénol) $>$ linalool (alcool) $>$ le camphre (cétone). L'hydrocarbure monoterpènique : $\alpha$-pinène est généralement moins toxique que les monoterpènes oxygénés.

Des observations similaires ont été faites par Pavela (2008), qui travaillant sur les effets des monoterpènes des huiles essentielles sur la mouche domestique Musca domestica L. (Diptera : Muscidae), a montré que l'efficacité de ces substances varie significativement selon le mode de leur application (par contact ou par inhalation). D'après ce même auteur, les monoterpènes les plus efficaces, une fois appliqués par contact, sont les phénols : le carvacrol et le thymol (DL 50 78 et $48 \mu \mathrm{g}$ /larve, respectivement). Par contre, dans le cas du traitement par inhalation, ce sont les hydrocarbures monoterpéniques dont notamment $\Upsilon$-terpinène et $\rho$-cymène $\left(\mathrm{DL}_{50} 64,73\right.$ et $74 \mu \mathrm{g} / \mathrm{cm}^{3}$, respectivement) qui sont les plus toxiques. Ces différences peuvent être dues en partie à la différence de volatilité et de dispersion des monoterpènes dans l'air (Pavela, 2008). D'une façon globale, nous montrons ici que, parmi les composés testés, ce sont les monoterpènes oxygénés notamment les phénols (thymol et carvacol) et alcools (linalool) qui sont les plus toxiques pour les adultes de l'acarien ravageur T. urticae. Pour les acariens et pour d'autres arthropodes aussi, plusieurs auteurs ont pu montrer la haute toxicité des monoterpènes oxygénés par rapport aux non oxygénés. Testant l'effet de ces composés sur les adultes du bruche du haricot Acanthoscelides obtectus (Coleoptera: Bruchidae), Regnault-Roger \& Hamraoui (1995) ont montré que les monoterpènes oxygénés (carvacrol, linalool et terpinéol) sont plus toxiques que les non oxygénés ( $\mathrm{p}$-cymène, cinnamaldéhyde, anéthole). Ceci serait dû aux différents modes d'action des différents constituants (Regnault-Roger \& Hamraoui, 1995). En effet, la différence de toxicité de ces monoterpènes peut être attribuée à la différence de leurs mécanismes d'action, de leur capacité de diffusion et de leur lipophilie. La différence des structures chimiques des monoterpènes est un autre facteur influençant leur efficacité.

Selon Hamdy et al. (2009), les hydrocarbures monoterpéniques, qu'ils soient cycliques ou acycliques sont les moins toxiques comme fumigants sur les femelles de l'acarien ravageur $T$. urticae. Dans notre cas aussi les hydrocarbures utilisés, tous cycliques, présentent, en général une activité acaricide assez modérée en comparaison avec les autres terpènes étudiés. Pour Hamdy et al. (2009) aussi, les hydrocarbures monoterpéniques sont moins efficaces entant qu'agent de contrôle de ces acariens que les monoterpènes oxygénés. Selon ces auteurs les monoterpènes à groupes fonctionnels oxygénés comme les phénols sont les plus toxiques sur les $T$. urticae. Ceci rejoint nos constats puisque le thymol et carvacrol ont été, quel que soit le mode de leur application, parmi les terpènes les plus toxiques. Les alcools terpéniques comme le linalool présentent aussi une activité prometteuse selon ces auteurs. Dans notre cas aussi la toxicité du linalool a été mise en évidence surtout lorsqu'il s'agit d'un traitement par contact.

En revanche, d'autres monoterpènes oxygénés (éther ou cétone) montrent, d'après notre étude, une toxicité largement moins importante que les phénols ou les alcools. Ainsi, 1.8-cineole, un éther monoterpénique, présente une bonne toxicité inhalatrice mais parait être peu toxique s'il est appliqué par contact. Les cétones monoterpéniques semblent aussi avoir une toxicité modérée, d'après nos résultats. C'est le cas du camphre, une cétone monoterpénique bicyclique qui, selon nos résultats, se classe parmi les monoterpènes les moins toxiques, qu'ils soient appliqués par inhalation ou par contact sur les adultes de l'acarien ravageur T. urticae. 
Le deuxième volet de notre étude vise à analyser les interactions entre 6 composés monoterpéniques associés en 15 combinaisons binaires différentes.

Nos résultats montrent que certains monoterpènes, notamment les phénols et alcools sont plus actifs lorsqu'ils sont appliqués sous forme de mélange que s'ils sont appliqués individuellement. À titre d'exemple, le carvacrol, un monoterpène phénolique, entraine $85 \%$ de mortalité lorsqu'il est appliqué individuellement contre une mortalité de $100 \%$ lorsqu'il est combiné à un autre monoterpène, qui dans ce cas, peut être un alcool (linalool), un éther (1,8cinéole), un hydrocarbure ( $\alpha$-pinène) ou encore une cétone (camphre). Des observations similaires ont été faites par Singh et al. (2009). Ces auteurs ont aussi montré que les alcools et phénols sont plus actifs en combinaison que lorsqu'ils sont pris individuellement.

Parmi les 15 combinaisons que nous avons testées, quatre se sont révélées synergiques, 10 additives et une seule antagoniste. Les 4 combinaisons synergiques sont les suivantes : (thymolcamphre); (linalool-camphre); (linalool- $\alpha$-pinène) et ( $\alpha$-pinène-thymol). Dans ce cas, l'efficacité des composés phénoliques (thymol) ou alcooliques (Linalool) se trouve améliorés lorsqu'ils sont combinés à des hydrocarbures comme l' $\alpha$-pinène ou à des cétones comme le camphre. À titre d'exemple, nos résultats montrent que le thymol interagit de manière synergique lorsqu'il est combiné à l' $\alpha$-pinène. Ceci se traduit par augmentation de la létalité de ces 2 composés envers les adultes de T. urticae: La mortalité observée, dans ce cas, est de $100 \%$ alors que celle attendue pour cette combinaison binaire n'est que de $78 \%\left(X^{2}=5.95\right.$; $\mathrm{Om}>\mathrm{E}$ ). Nos résultats s'accordent avec ceux Tak \& Isman (2017). Ces auteurs ont étudié l'effet synergique des monoterpènes sur la fausse-arpenteuse du chou Trichoplusia ni (Lepidoptera : Noctuidae). Ils ont pu montrer l'existence d'une forte interaction synergique entre le thymol et d'autres hydrocarbure comme l' $\alpha$-terpinène (Tak $\&$ Isman, 2017). Au total, la synergie qui se produit entre certains composants des huiles essentielles végétales serait capable de produire une nette amélioration de la bioactivité de ces derniers : ces composés monoterpéniques sont considérablement plus toxiques s'ils sont associés en combinaisons binaires que s'ils sont appliqués individuellement. De tels effets sont courants pour les terpènes, qui, étant des composés hydrophobes, présentent des effets synergiques avec d'autres composants en les solubilisant et en facilitant leur dispersion à travers les membranes cellulaires (Rattan, 2010). De tels effets augmentent l'efficacité de ces composés, permettant leur utilisation en plus petites quantités dans un mélange pour atteindre des niveaux d'efficacité satisfaisants.

Parmi les 15 combinaisons binaires testées, 10 combinaisons sont classées comme étant à effet additif. Dans ce cas, l'effet létal combiné de deux composés terpéniques représente la somme des effets de chaque composé pris individuellement. Les combinaisons à effet additif trouvées sont les suivantes : (thymol-1,8-cinéole); (carvacrol-1,8-cinéole); (thymol-linalool) ; (thymolcarvacrol) ; (carvacrol-linalool) ; (linalool-1,8-cinéole); (carvacrol- $\alpha$-pinène); (carvacrolcamphre) ; (1,8-cinéole-camphre) ; ( $\alpha$-pinène-camphre). Dans ce cas, l'association de deux monoterpènes (éther, cétone, alcool, hydrocarbure ou un phénol) produit le même effet toxique sur les acariens que la somme des effets de chaque composé pris individuellement. À titre d'exemple, le mélange binaire (linalool-1,8-cinéole) entraine une mortalité observée de 100\%, statistiquement similaire à celle attendue si on considère la somme des effets de ces 2 composés 
pris séparément $(91.25 \%)\left(\mathrm{X}^{2}=0.912 ; \mathrm{Om}>\mathrm{E}\right)$. Nos résultats concordent avec ceux Singh et al. (2009) qui ont étudié la toxicité de certains constituants des HEs et leurs mélanges binaires sur Chilo partellus (Lepidoptera: Pyralidae). Ces auteurs ont pu montrer l'existence d'interaction additive entre 1,8-cinéole et le linalool.

Nos résultats montrent, enfin, que la seule combinaison binaire à effet antagoniste est celle entre l'hydrocarbure monoterpénique: $\alpha$-pinène et l'éther: 1,8-cinéole. Dans ce cas, le mélange binaire évalué présente un effet antagoniste puisque l'effet de ces 2 composés purs dépasse celui de ces mêmes composés associés en combinaison binaire $\left(\mathbf{X}^{\mathbf{2}}=\mathbf{1 0 . 3 5} ; \mathbf{O m}<\mathbf{E}\right)$. Cela montre que la combinaison de deux substances différentes dans un mélange binaire n'est pas toujours avantageuse, autrement dit l'activité acaricide de certains composés peut être considérablement atténuée s'ils sont mélangés ensemble dans une combinaison binaire. Nos observations rejoignent ceux de Sharma et al (2019) pour qui l'effet antagoniste indique que les composés mélangés dans une combinaison sont moins actifs que lorsqu'ils sont à l'état pur. L'antagonisme de certaines combinaisons de monoterpènes pourrait être dû au comportement d'un composé par rapport l'autre composé : l'un des composés peut en effet agir en modifiant le taux d'absorption du second composé, sa distribution, son métabolisme ou son excrétion par le ravageur (Tannenbaum et al., 2014). L'antagonisme pourrait aussi être dû à la compétition entre les composés pour accéder à un même récepteur cellulaire ou à une même cible biochimique ou physiologique chez l'acarien. Dans notre cas, la combinaison associant 1,8cinéole à l' $\alpha$-pinène a entraîné une réponse antagoniste qui pourrait s'expliquer par leurs mécanismes d'actions sur les sites cibles (Sikkema et al., 1994 ; Savelev et al., 2003). Comme les deux composés sont des monoterpènes cycliques, ils peuvent avoir le même récepteur cellulaire ou à la même cible biochimique ou physiologique chez l'acarien. Pour se lier à cette cible, les 2 composés doivent entrer en compétition, ce qui atténue la toxicité globale de la combinaison binaire testée vis-à-vis de l'acarien T. urticae. Par conséquent, les composés combinés dans un même mélange binaire doivent avoir des cibles biochimiques ou physiologiques différentes dans l'arthropode traité. Ceci permettrait un meilleur contrôle de ce dernier.

\section{Conclusion}

Certains monoterpènes peuvent être retenus pour le développement de mélanges binaires à haut potentiel acaricide. Compte tenu de nos résultats, des combinaisons binaires à effet synergique associant le phénol (carvacrol et le thymol) ou l'alcool (linalool) à un hydrocarbure ou à une cétone peuvent être recommandées au vu de leur potentiel acaricide élevé. Rechercher des associations synergiques permettrait aussi de réduire les doses efficaces des composés dans les mélanges sans que ces derniers perdent de leur d'efficacité. Ceci peut servir de base au développement de nouvelles formulations à la faible teneur en principes actifs grâce à des associations judicieuses de substances. 


\section{Références}

Abbott W.S., 1925. A method for computing the effectiveness of an insecticide. J. Econ. Entomol., 18, 265-267.

Abdelgaleil S.A.M., Badawy M. E.I. Mahmoud N. F. \& Marei Abd El-Salam M. 2019. Acaricidal activity, biochemical effects and molecular docking of some monoterpenes against two-spotted spider mite (Tetranychus urticae Koch). Pesticide Biochemistry and Physiology. 156, 105-115.

Andrade-Ochoa S., Correa-Basurto J., Rodríguez-Valdez L. M., et al., 2018. In vitro and in silico studies of terpenes, terpenoids and related compounds with larvicidal and pupaecidal activity against Culex quinquefasciatus Say (Diptera: Culicidae). Chemistry Central Journal, 12, 53.

Attia S., Grissa K.L., Lognay G., Heuskin S., Mailleux A.C. \& Hance T., 2011. Chemical composition and acaricidal properties of Deverra scoparia essential oil (Araliales: Apiaceae) and blends of its major constituents against Tetranychus urticae (Acari: Tetranychidae). J. Econ. Entomol., 104, 1220-1228.

Attia S., Grissa K.L., Ghrabi Z.G., Mailleux A.C., Lognay G. \& Hance T., 2012. Acaricidal activity of 31 essential oils extracted from plants collected in Tunisia. Journal of Essential Oil, 24, 279-288.

Attia S., Grissa K., Lognay G., Bitume E., Hance T. \& Mailleux A.C., 2013. A review of the major approaches to control the worldwide pest Tetranychus urticae (Acari: Tetranychidae) with special references to natural pesticides. J. Pest Sci., 86, 361-386.

Badawy M.E.I., El-Arami S.A.A. \& Samir A. A., 2010. Acaricidal and quantitative structure activity relationship of monoterpenes against the two-spotted spider mite, Tetranychus urticae. Experimental \& Applied acarology, 52, 261-274.

Bounatirou S., Smiti S., Miguel M.G., Rejeb M.N., Neffati M., Costa M.M., Faleiro L, Figueiredo A.C., Barroso J.G. \& Pedro L.G., 2007. Chemical composition, antioxidant and antibacterial activities of the essential oils isolated from Tunisian Thymus capitatus Hoff. Et Link. Food Chem., 105, 146-155.

Butt T.M. \& Goettel M., 2000. Bioassays of Entomopathogenic fungi. In: Bioassays of Entomopathogenic microbes and nematodes (ed. Navon, A. and Ascher, K.R.S.) CAB International, Wallingford, Oxon, U.K. Chapter 4. 141-195.

Carović-Stanko K., Orlić S., Politeo O. \& Šatović Z., 2010. Composition and antibacterial activities of essential oils of seven Ocimum taxa. Food Chemistry, 119, 196-201.

Cavalcanti S.C.H., Niculau Edos S., Blank A.F., Camara C.A.G., Araujo I.N. \& Alves P.B., 2010. Composition and acaricidal activity of Lippia sidoides essential oil against twospotted spider mite (Tetranychus urticae Koch). Bioresour Technol., 101,829-832.

Deletre E., Chandre F., Williams L., Duménil C., Menut C. \& Martin T., 2015. Electrophysiological and behavioral characterization of bioactive compounds of the Thymus vulgaris, Cymbopogon winterianus, Cuminum cyminum and Cinnamomum zeylanicum essential oils against Anopheles gambiae and prospects for their use as bednet treatmen. Parasites Vectors, 8, 316. 
Dias C.N. \& Moraes D.F.C., 2014. Essential oils and their compounds as Aedes aegypti L. (Diptera: Culicidae) larvicides: review. Parasitol. Res. 113, 565-592.

Finney DJ., 1971. Probit analysis, 3rd edn. Cambridge University Press, Cambridge

Hamdy E., Ayad A. \& El-Sebae A kh H. (2009): Acaricidal activity of plant extracts and their main terpenoids on the twospotted spider mite Tetranychus urticae. Alexandria Science Exchange Journal, 30, 344-349.

Herman A., Tambor K \& Herman A., 2016. Linalool Affects the Antimicrobial Efficacy of Essential Oils. Current Microbiology, 72, 165-172.

Herron G.A. \& Rophail J., 2003. First detection of chlorfenapyr ( $\mathrm{Sec}$ ) resistance in two-spotted spider mite (Acari:Tetranychidae) from nectarines in an Australia orchard. Exp Appl Acarol., 31, 131-134.

Hummelbrunner L. \& Isman M., 2001. Acute, sublethal, antifeedant, and synergistic effects of monoterpenoid essential oil compounds on the tobacco cutworm, Spodoptera litura (Lep., Noctuidae), J. Agric. Food Chem., 49, 715-720.

Isman M.B., 2001. Pesticides based on plant essential oils for management of plant pests and diseases. In: International symposium on development of natural pesticides from forest resources. Korea Forest Reasearch Institute, Seoul, Korea, 1-9.

Ketoh G.K., Koumaglo H.K., Glitho I.A. \& Huignard J., 2006. Comparative effects of Cymbopogon schoenanthus essential oil and piperitone on Callosobruchus maculates development. Fitoterapia, 77, 506-510.

Kim E.H., Kim H.K. \& Ahn Y.J., 2003. Acaricidal activity of clove bud oil compounds against Dermatophagoides farinae and Dermatophagoides pteronyssinus (Acari: Pyroglyphidae). J Agric Food Chem., 51, 885-889.

Kim S.I., Yi J.H., Tak J.H. \& Ahn Y.J., 2004. Acaricidal activity of plant essential oils against Dermanyssus gallinae (Acari: Dermanyssidae). Veterinary Parasitology, 120, 297-304.

Kim Y.J., Lee Si.H., Lee Si-W., Ahn Y.J., 2004. Fenpyroximate resistance in Tetranychus urticae (Acari: Tetranychidae): cross-resistance and biochemical resistance mechanisms. Pest Manag Sci., 10, 1001-1006.

Kim Y.J., Park H.M., Cho J.R. \& Ahn Y.J., 2006. Multiple resistance and biochemical mechanisms of pyridaben resistance in Tetranychus urticae (Acari: Tetranychidae). J Econ Entomol., 99, 954-980.

Kim S.W., Lee H.R., Jang M.J., Jung C.S. \& Park I.K., 2016. Fumigant toxicity of lamiaceae plant essential oils and blends of their constituents against adult rice weevil Sitophilus oryzae. Molecules, 21, 361.

Koul O., Singh R., Kaur B. \& Kanda D., 2013. Comparative study on the behavioral response and acute toxicity of some essential oil compounds and their binary mixtures to larvae of Helicoverpa armigera, Spodoptera litura and Chilo partellus. Industrial Crops and Products, 49, 428-436.

Lagziri M., 2013. Étude de l'effet des produits phytosanitaires sur les acariens ravageurs et leurs ennemis naturels : Cas du fraisier du Loukkos. Thèse de doctorat, Discipline: Protection des Plantes, Spécialité: Acarologie, Université Abdelmalek Essaadi Faculté Des Sciences Et Techniques - Tanger. 249pp. 
Lagziri M., Benicha M., M'rabet R. \& El Amrani A., 2015. Influence de l'usage préventif des pesticides sur les acariens Tetranychus urticae et Phytoseiulus persimilis (Acari : Tetranychidae, Phytoseiidae) présents en cultures de fraisiers du Nord du Maroc», BASE, 19, 355-363.

Lee S., Tsao R., Peterson Ch. \& Coats J., 1997. Insecticidal activity of monoterpenoids to western corn rootworm (Coleoptera: Chrysomelidae), twospotted spider mite (Acari: Tetranychidae), and house fly (Diptera: Muscidae), J. Econ. Entomol., 90, 883-892.

Lindberg C., Melathopoulus A. \& Winston M., 2000. Laboratory evaluation of miticides to control Varroa jacobsoni, a honey bee parasite. J. Econ. Entomol., 93, 189-198.

Marquier M. \& Arnault V., 2008. Evaluation de l'acarien prédateur phytoseiulus persimilis pour contrôler l'acarien jaune Tetranychus urticae sur fraisiers à l'ile de la réunion. AFPP -8 ème Conférence internationale sur les ravageurs en agriculture Montpellier. 615-680.

Miresmailli S., Bradbury R. \& Isman M.B., 2006. Comparative toxicity of Rosmarinus officinalis L. essential oil and blends of its major constituents against Tetranychus Urticae Koch (Acari: Tetranychidae) on two different host plants. Pestmanagement Science. 62, $366-371$.

Ngamo T.S.L., Ngatanko I., Ngassoum M.B., Mapongmestsem P.M. \& Hance T., 2007. Persistence of insecticidal activities of crude essential oils of three aromatic plants towards four major stored product insect pests. African Journal of Agricultural Research, 2, 173177.

Overmeer W.P.J., 1967. Genetics of resistance to tedion in Tetranychus urticae (Koch) Arch. Neerl. Zol., 17, 296-349.

Pandey S.K., Tandon S., Ahmad A., Singh A.K. \& Tripathi A.K., 2013. Structure-activity relationships of monoterpenes and acetyl derivatives against Aedes aegypti (Diptera: Culicidae) larvae. Pest Manag Sci. doi:10.1002/ps.3488

Papachristos D.P., Karamanoli K.I., Stamopoulos D.C. \& Menkissoglu-Spiroudi U., 2004. The relationship between the chemical composition of three essential oils and their insecticidal activity against Acanthoscelides obtectus (Say). Pest Manag Sci., 60 (5):514-520.

Pavela R., 2008. Insecticidal properties of several essential oils on the house fly (Musca domestica L.). Phytotheropia Res., 22, 274-278.

Pavela R., 2015. Acute toxicity and synergistic and antagonistic effects of the aromatic compounds of some essential oils against Culex quinquefasciatus Say larvae. Parasitol. Res., 114, 3835-3853.

Pavela R., Shchenikova A., Stepanycheva E., Petrova M. \& Chermenskaya T., 2016. Essential oils as prospective fumigants against Tetranychus urticae Koch. Industrial Crops and Products, 94, 755-761.

Rabiou I., 2019. Liste des produits insecticides et acaricides en vente ou utilisés au Niger en 2019. Réseau National des Chambres d'Agriculture du Niger. 1-15.

Rattan S.R., 2010. Mechanism of action of insecticidal secondary metabolites of plant origin. Review, Crop Protection, 29 (9), 913-920. 
Regnault R.C. \& Hamraoui A., 1995. Fumigant toxic activity and reproductive inhibition induced by monoterpenes on Acanthoscelides obtectus (Say) (Coleoptera), a bruchid of kidney bean (Phaseolus vulgaris L.). J. Stored Prod. Res. 31, 291-299.

Regnault-Roger C., Philogène B.J.R. \& Vincent C. 2002. De nouveaux phytoinsecticides pour le troisième millénaire? Dans : Biopesticides d'origine végétale. Tec et Doc (eds.), Paris, $19-40$.

Reis S.L., Mantello A.G., Rossete E.A.G., Cardoso A.M. \& Beleboni R.O., 2014. Insecticidal and repellent activity of typical monoterpenes from plant essential oils against Callosobruchus maculatus (Fabr. 1775). BMC Proceedings, 8(Suppl 4): P115.

http://www.biomedcentral.com/1753-6561/8/S4/P11

Santos S.R.L., Silva V.B., Melo M.A., Barbosa J.D.F., Santos R.L.C., Sousa D.P. \& Cavalcanti S.C.H., 2010. Toxic effects on and structure-toxicity relationships of phenylpropanoids, terpenes, and related Compounds in Aedes aegypti larvae. Vector-Borne Zoonotic Dis. doi: 10.1089/vbz.2009.0158.

Savelev S., Edward J Okello. Perry N.S.L. et al., 2003. Synergistic and antagonistic interactions of anticholinesterase terpenoides in Salvia lavandulaefolia essential oil. Pharmacology, Biochemistry and Behavior, 75(3), 661-668.

Seo S.M., Jung C.S., Kang J., Lee H.R., Kim S.W., Hyun J. \& Park I.K., 2015. Larvicidal and acetylcholinesterase inhibitory activities of Apiaceae plant essential oils and their constituents against Aedes albopictus and formulation development. J. Agric. Food Chem., 63, 9977-9986.

Sharma A., Singh H.P., Batish D.R. \& Kohli R.K., 2019. Chemical profiling, cytotoxicity and phytotoxicity of foliar volatiles of Hyptis suaveolens. Ecotoxicol Environ Safe, 171, 86370 .

Sikkema J., Debont J. \& Poolman, B., 1994. Interactions of Cyclic Hydrocarbons with Biological Membranes. The Journal of Biological Chemistry, 269(11), 8022-8028.

Singh D. \& Singh A. K., 1991. Repellent and Insecticidal Properties of Essential Oils Against Housefly, Musca Domestica L. International Journal of Tropical Insect Science, 12, 487 491.

Singh R., Rup P.J. \& Koul O., 2008. Bioefficacy of 1.8-cineole from Eucalyptus camaldulensis var. obtusa and linalool from Luvanga scandans against Spodoptera litura (Lepidoptera: Noctuidae) and combination effects with some other monoterpenoids. BiopesticideInternational, 4, 128-137.

Singh R., Koul O, Rup P.J. \& Jindal J., 2009. Toxicity of some essential oil constituents and their binary mixtures against Chilo partellus (Lepidoptera: Pyralidae). International Journal of Tropical Insect Science, 29, 93-101.

Tak J.H. \& Isman M.B., 2017. Acaricidal and repellent activity of plant essential oil-derived terpenes and the effect of binary mixtures against Tetranychus urticae Koch (Acari: Tetranychidae). Industrial Crops and Products, 108, 786-792.

Tanenbaum M.E., Gilbert L.A., Qi L.S., Weissman J.S. \& Vale R.D., 2014. A protein-tagging system for signal amplification in gene expression and fluorescence imaging. Cell, 159(3), 635-46. 
Tripathi A.K., Prajapati V. \& Kumar S., 2003. Bioactivity of 1-carvone, d-carvone and dihydrocarvone towards three stored product beetles. J. Econ. Entomol., 96, 1594-1601.

Van Leeuwen T., Stillatus V. \& Tirry L., 2004. Genetic analysis and cross-resistance spectrum of a laboratoryselected chlorfenapyr resistant strain of two-spotted spider mite (Acari: Tetranychidae). Exp Appl Acarol., 32, 249-261.

Watanabe N., Watanabe S., Nakajima R., Moon J.H., Shimokihara K., Inagaki J., Etoh H., Asai T., Skata R \& Ina K., 1993. Formation of flower fragrance compounds from their precursors By enzymic action during flower opening. Biosci. Biotech. Biochem., 57, 1101-1106.

Wu L., Huo X., Zhou X., Zhao D., He W., Liu S., Liu H., Feng T. \& Wang C., 2017. Acaricidal Activity and Synergistic Effect of Thyme Oil Constituents against Carmine Spider Mite Tetranychus Cinnabarinus (Boisduval). Molecules, 22, 1-12.

Zhu B.C., Henderson G., Chen F., Maistrello L. \& Laine R.A., 2001. Nootkatone is a repellent for Formosan subterranean termite (Coptotermes formosanus). J Chem Ecol, 27(3), 52331 . 